Georgian Mathematical Journal

Volume 8 (2001), Number 2, 275-281

\title{
PROPER MOVING AVERAGE REPRESENTATIONS AND OUTER FUNCTIONS IN TWO VARIABLES
}

\author{
L. GAWARECKI, V. MANDREKAR, AND P. RICHARD
}

\begin{abstract}
In this work, we consider the problem of moving average representations for random fields. As in the Kolmogorov-Wiener case, such representations lead to interesting questions in harmonic analysis in the polydisc. In particular, we study outer functions with respect to half-space, semigroup and quarterplane and their interrelations.
\end{abstract}

2000 Mathematics Subject Classification: 42B30, 60G10, 60G60.

Key words and phrases: Outer functions, proper moving average representations, stationary random fields.

\section{INTRODUCTION}

Professor N. Vakhania has been interested in the area of Prediction Theory and Analysis for infinite dimensional stationary processes, [1]. In this work we study the moving average (MA) representations for weakly stationary random fields under half-spaces, semigroups and quarter-planes. We relate the properness of the MA representation to the analytic properties of the factor of the spectral density. We interpret the results of Helson and Lowdenslager, [3], in this context, and relate them to the $H$-outer property. Using a result in [3], one can show the equivalence of the outer property of the factor, and the properness of the semigroup induced MA representation. We use this result to relate $\lambda$ outer functions (in one variable, with the other variable acting as a parameter), and half-space MA representations.

Finally, we take up the quarter-plane MA representation and relate its properness to $H$-outer property of the factor. This can be exploited to obtain a probabilistic result of Soltani, [9]. Connection between outer functions in the sense of [3] and outer functions in $H^{2}\left(T^{2}\right)$ is used to obtain the analytic results of Izuchi and Matsugu, [5], with very simple proofs. Throughout the paper, we use the standard terminology of the book [2].

\section{Half-Space MA Representations}

Let $\{\Omega, \mathcal{F}, P\}$ be a probability space, and $L^{2}(\Omega, \mathcal{F}, P)$ be the complex Hilbert space of (equivalence classes of) $P$-square integrable complex-valued functions. A family $\left\{X_{t}, t \in \mathbf{Z}^{d}\right\} \subset L^{2}(\Omega, \mathcal{F}, P)$ is called a weakly stationary random field 
if $E\left(X_{t}\right)=c($ from now on $c=0)$, and

$$
E\left(X_{t} X_{t^{\prime}}\right)=\mathcal{R}\left(t-t^{\prime}\right)
$$

In the case of $d=1,\left\{X_{n}, n \in \mathbf{Z}\right\}$ is called a weakly stationary process. With $\left\{X_{n}\right\}$, one associates a spectral measure $F$ through the Bochner Theorem,

$$
\mathcal{R}(n)=\frac{1}{2 \pi} \int_{-\pi}^{\pi} e^{i n \lambda} d F(\lambda) .
$$

A stationary process has a $\mathbf{M A}$ representation if

$$
X_{n}=\sum_{k=0}^{\infty} a_{k} \xi_{n-k},
$$

where $\left\{\xi_{k}, k \in \mathbf{Z}\right\}$ are orthonormal elements of $L^{2}(\Omega, \mathcal{F}, P)$. It is well known by a result of Kolmogorov and Wiener, that $X_{n}$ has a MA representation if and only if $F \ll$ Leb, and the density is given by $f\left(e^{i \lambda}\right)=\left|\varphi\left(e^{i \lambda}\right)\right|^{2}$, where $\varphi\left(e^{i \lambda}\right)=\sum_{k=0}^{\infty} \bar{a}_{k} e^{i k \lambda}$.

From the point of view of prediction, one needs to know when the MA representation (2.1) is proper, i.e., when

$$
H(X: n)=H(\xi: n), \text { for each } n \text {. }
$$

Here, $H(X: n)=\overline{\operatorname{sp}}\left\{X_{k}, k \leq n\right\}$.

It is obvious that the above condition holds true if and only if $\varphi\left(e^{i \lambda}\right)$ is $H$ outer, i.e., $\overline{\operatorname{sp}}\left\{e^{i n \lambda} \varphi\left(e^{i \lambda}\right): n \geq 0\right\}=H^{2}(T)$, where $H^{2}(T)=\overline{\operatorname{sp}}\left\{e^{i n \lambda}, n \geq 0\right\}$, and the closure refers to the space $L^{2}([-\pi, \pi]$, Leb). An analytic condition for $\varphi$ to be $H$-outer is given by

$$
\log \left|\int_{-\pi}^{\pi} \varphi\left(e^{i \lambda}\right) d \sigma\right|=\int_{-\pi}^{\pi} \log \left|\varphi\left(e^{i \lambda}\right)\right| d \sigma,
$$

where $\sigma$ is the normalized Lebesgue measure on $[-\pi, \pi]$.

In case $G=\mathbf{Z}^{2}$, Helson and Lowdenslager ([3]) considered the analogue of this problem by putting an ordering on $\mathbf{Z}^{2}$ induced by a semigroup $S$ so that $S \cup-S=\mathbf{Z}^{2}$, and $S \cap-S=\{(0,0)\}$. A particular example of $S$ is

$$
S=\left\{(j, k): j \in \mathbf{Z}_{+} \text {for } k=0 \text { and } j \in \mathbf{Z} \text { for } k \geq 1\right\} .
$$

They showed that a stationary random field has a MA representation, i.e.,

$$
X_{m, n}=\sum_{(j, k) \in S} a_{j, k} \xi_{m-j, n-k}
$$

with $\left\{\xi_{j, k}\right\}_{(j, k) \in \mathbf{Z}^{2}}$, orthonormal elements in $L^{2}(\Omega, \mathcal{F}, P)$ if and only if the spectral measure of $\left\{X_{m, n}\right\}$ on $T^{2}$ is absolutely continuous with respect to $\sigma_{2}$, the normalized Lebesgue measure on $T^{2}$, and $f\left(e^{i \lambda}, e^{i \mu}\right)=\left|\varphi\left(e^{i \lambda}, e^{i \mu}\right)\right|^{2}$. Here $\varphi\left(e^{i \lambda}, e^{i \mu}\right)=\sum_{(j, k) \in S} \bar{a}_{j, k} e^{i j \lambda+i k \mu}$ is of analytic type (in the sense of $S$ ). 
Using the ordering on $\mathbf{Z}^{2}$ induced by $S$, i.e., $(j, k)<\left(j^{\prime}, k^{\prime}\right)$ if $\left(j^{\prime}-j, k^{\prime}-k\right) \in$ $S$, we define

$$
H(X:(m, n))=\overline{\operatorname{sp}}\left\{X_{j, k}:(j, k)<(m, n) \in \mathbf{Z}^{2}\right\} .
$$

We say that a MA $S$-representation is proper if

$$
H(X:(m, n))=H(\xi:(m, n)) \text {, for all }(m, n) \in \mathbf{Z}^{2} .
$$

From the result in [3] we get that a MA representation is proper if and only if

$$
\log \left|\int_{T^{2}} \varphi\left(e^{i \lambda}, e^{i \mu}\right) d \sigma_{2}\right|=\int_{T^{2}} \log \left|\varphi\left(e^{i \lambda}, e^{i \mu}\right)\right| d \sigma_{2},
$$

where $\varphi$ is the function of $S$-analytic type associated with the MA $S$-representation. A function $\varphi$ of $S$-analytic type is called outer if it satisfies equation (2.4).

Given a set $A \subset \mathbf{Z}^{2}$, and $g \in L^{2}\left(T^{2}, \sigma_{2}\right)$, denote $[A]:=\overline{\operatorname{sp}}\left\{e^{i j \lambda+i k \mu}:(j, k) \in A\right\}$, and $[g]_{A}:=\overline{\operatorname{sp}}\left\{e^{i j \lambda+i k \mu} g:(j, k) \in A\right\}$, closed linear subspaces of $L^{2}\left(T^{2}, \sigma_{2}\right)$. The following result is a consequence of Theorem 6 in [3].

Theorem 2.1. Let $\varphi$ be of S-analytic type. Then the following statements are equivalent:

(i) $\varphi$ is outer,

(ii) $[\varphi]_{S}=[S]$.

Let us denote by $A_{\lambda}:=\mathbf{Z} \times \mathbf{Z}_{+}$the half-space in $\mathbf{Z}^{2}$, and $H_{\lambda}^{2}:=\left[A_{\lambda}\right]$. The condition (ii) of Theorem 2.1 implies that an outer function $\varphi$ of $S$-analytic type satisfies

$$
H_{\lambda}^{2}=\left[A_{\lambda}\right]=[[S]]_{A_{\lambda}}=\left[[\varphi]_{S}\right]_{A_{\lambda}}=[\varphi]_{A_{\lambda}},
$$

giving $[\varphi]_{A_{\lambda}}=H_{\lambda}^{2}$.

Following [5], we define a function $g \in L^{2}\left(T^{2}, \sigma_{2}\right)$ as $\lambda$-outer if the cut function $g_{\lambda}\left(e^{i \mu}\right)=g\left(e^{i \lambda}, e^{i \mu}\right)$ is outer in the variable $e^{i \mu}, \sigma$-a.e. (in $\left.e^{i \lambda}\right)$.

The following lemma is a consequence of a result in [4].

Lemma 2.2. If $g \in H_{\lambda}^{2}$, then $g$ is $\lambda$-outer if and only if $[g]_{A_{\lambda}}=H_{\lambda}^{2}$.

Proof. By Theorem 2 in [4], $[g]_{A_{\lambda}}=q H_{\lambda}^{2}$, where $q$ is a unimodular function, such that the cut function $q_{\lambda}$ of $q$ is inner in $e^{i \mu}$. Since $g=q h$, and the cut function of $g$ is outer, we get that $q$ is constant in $e^{i \mu}, \sigma$-a.e. Thus $q H_{\lambda}^{2}=H_{\lambda}^{2}$, giving the necessity.

To get the sufficiency, we assume without lost of generality that $g\left(e^{i \lambda}, e^{i \mu}\right)$ is in $H^{2}\left(T_{\mu}\right)$ for all $\lambda$, and as in [2], for each $\lambda$, we define

$$
G_{r}\left(e^{i \lambda}, e^{i \mu}\right)=\exp \left(\int_{T} \frac{e^{i \lambda^{\prime}}+r e^{i \mu}}{e^{i \lambda^{\prime}}-r e^{i \mu}} \log \left|g\left(e^{i \lambda}, e^{i \mu}\right)\right| d \sigma\left(\lambda^{\prime}\right)\right) .
$$


Then for all $e^{i \lambda}, \lim _{r \rightarrow 1^{-}} G_{r}\left(e^{i \lambda}, e^{i \mu}\right)=G\left(e^{i \lambda}, e^{i \mu}\right)$ is an outer factor (in $\left.e^{i \mu}\right)$ of $g$ as a function of $e^{i \mu}$. Since

$$
\int_{T^{2}}|G|^{2} d \sigma_{2}=\int_{T^{2}}|g|^{2} d \sigma_{2}<\infty
$$

the function $G \in L^{2}\left(T^{2}, \sigma_{2}\right)$, we obtain that $G$ is $\lambda$-outer. Then $q=g / G$ is inner in $e^{i \mu}$ for all $\lambda$. Hence, $[g]_{A_{\lambda}}=q[G]_{A_{\lambda}}$. From the proof of the necessity, $[G]_{A_{\lambda}}=H_{\lambda}^{2}$. Thus $H_{\lambda}^{2}=[g]_{A_{\lambda}}=q H_{\lambda}^{2}$ and consequently, for each $\lambda, q$ is constant in $\mu$. Thus $g=q G$ is $\lambda$-outer.

As a consequence, we obtain the following theorem.

Theorem 2.3. Let $g$ be of $S$-analytic type. If $g$ is outer, then $g$ is $\lambda$-outer.

Given a function $g$ of $S$-analytic type, we have

$$
g\left(e^{i \lambda}, e^{i \mu}\right)=\sum_{j=0}^{\infty} a_{j, 0} e^{i j \lambda}+\sum_{k \geq 1} \sum_{j \in \mathbf{Z}} a_{j, k} e^{i j \lambda+i k \mu}
$$

Let

$$
h\left(e^{i \lambda}\right)=\sum_{j=0}^{\infty} a_{j, 0} e^{i j \lambda}
$$

and observe that

$$
\int_{T} g\left(e^{i \lambda}, e^{i \mu}\right) d \sigma(\mu)=h\left(e^{i \lambda}\right) .
$$

Thus if $h$ is outer, then

$$
\begin{aligned}
\log \left|\int_{T^{2}} g\left(e^{i \lambda}, e^{i \mu}\right) d \sigma_{2}\right| & =\log \left|\int_{T} \int_{T} g\left(e^{i \lambda}, e^{i \mu}\right) d \sigma(\mu) d \sigma(\lambda)\right| \\
& =\int_{T} \log \left|\int_{T} g\left(e^{i \lambda}, e^{i \mu}\right)\right| d \sigma(\lambda) .
\end{aligned}
$$

If further $g$ is $\lambda$-outer, then $\log \left|\int_{T} g\left(e^{i \lambda}, e^{i \mu}\right) d \sigma(\mu)\right|=\log \int_{T}\left|g\left(e^{i \lambda}, e^{i \mu}\right)\right| d \sigma(\mu)$. Hence we get

Lemma 2.4. Let $g$ be of $S$-analytic type, and $h$ be as in (2.5). If $h$ is outer and $g$ is $\lambda$-outer, then $g$ is outer. 


\section{Quarterplane MA Representations}

In [6], a quarterplane MA (QMA) representation was studied for $G=\mathbf{Z}^{2}$. Here, one does not have an ordering on $\mathbf{Z}^{2}$ (except lexicographic). We say that $\left\{X_{m, n},(m, n) \in \mathbf{Z}^{2}\right\}$ has a QMA representation if

$$
X_{m, n}=\sum_{k \geq 0} \sum_{j \geq 0} a_{j, k} \xi_{m-j, n-k},
$$

where $\left\{\xi_{j, k},(j, k) \in \mathbf{Z}^{2}\right\}$ is an orthonormal family. We say that a function $\varphi \in$ $H^{2}\left(T^{2}\right)$ if

$$
\varphi\left(e^{i \lambda}, e^{i \mu}\right)=\sum_{j \geq 0} \sum_{k \geq 0} b_{j, k} e^{i j \lambda+i k \mu} .
$$

We note that $\varphi \in H^{2}\left(T^{2}\right)$ implies that $\varphi$ is of $S$-analytic type. It is easy to verify that $\left\{X_{m, n}\right\}$ has the QMA representation (3.1) if and only if its spectral measure $F \ll \sigma_{2}$, and the density $f\left(e^{i \lambda}, e^{i \mu}\right)=\left|\varphi\left(e^{i \lambda}, e^{i \mu}\right)\right|^{2}$, where $\varphi \in H^{2}\left(T^{2}\right)$ and

$$
\varphi\left(e^{i \lambda}, e^{i \mu}\right)=\sum_{j, k \geq 0} \bar{a}_{j, k} e^{i j \lambda+i k \mu} .
$$

A QMA representation is proper if and only if

$$
H(X:(m, n))=\overline{\mathrm{sp}}\left\{X_{j, k}: j \leq m, k \leq n\right\}=H(\xi:(m, n)) .
$$

For any second order random field $\left\{y_{m, n}:(m, n) \in \mathbf{Z}^{2}\right\}$, we denote

$$
\begin{aligned}
L^{1}(y: m) & =\overline{\mathrm{sp}}\left\{y_{j, k}: j \leq m, k \in \mathbf{Z}\right\} \\
\text { and } L^{2}(y: n) & =\overline{\operatorname{sp}}\left\{y_{j, k}: j \in \mathbf{Z}, k \leq n\right\} .
\end{aligned}
$$

Also, let $p^{i}(y: m)$ be the projection onto $L^{i}(y: m), i=1,2$. We shall drop the dependence on $y$ when it is clear from the context. We observe that for $\left\{\xi_{j, k},(j, k) \in \mathbf{Z}^{2}\right\}$,

$$
p^{1}(m) p^{2}(n)=p(m, n),
$$

where $p(m, n)$ is the projection on $H(\xi:(m, n))$. Thus we obtain that if the QMA representation (3.1) is proper, i.e., $H(X:(m, n))=H(\xi:(m, n))$, then for the process $\left\{X_{m, n}:(m, n) \in \mathbf{Z}^{2}\right\}$, equality (3.3) holds true. This condition was introduced in [6]. Denote, as in [6], $L^{i}(X:-\infty)=\bigcap_{m} L^{i}(X: m), i=1,2$. It was further proved in [6] that under condition (3.3), and the condition:

$$
\overline{\mathrm{sp}}\left\{L^{1}(X:-\infty) \cup L^{2}(X:-\infty)\right\}=\{0\},
$$

a weakly stationary random field has a proper QMA representation.

We observe

Theorem 3.1. The $\boldsymbol{Q M A}$ representation (3.1) for $\left\{X_{m, n}\right\}$ is proper if and only if the following three conditions are satisfied:

(i) the spectral measure, $F$, of $\left\{X_{m, n}\right\}$, satisfies $F \ll \sigma_{2}$,

(ii) the density $f\left(e^{i \lambda}, e^{i \mu}\right)=\left|\varphi\left(e^{i \lambda}, e^{i \mu}\right)\right|^{2}$, with $\varphi \in H^{2}\left(T^{2}\right)$, 
(iii) $[\varphi]_{\mathbf{Z}_{+}^{2}}=H^{2}\left(T^{2}\right)$.

From the above theorem, we obtain the following result of Soltani [9].

Theorem 3.2. A second order stationary random field has a proper $\boldsymbol{Q M A}$ representation if and only if it satisfies conditions (3.3), (3.4), and (i)-(iii) of Theorem 3.1.

Since $\varphi \in H^{2}\left(T^{2}\right)$, with $[\varphi]_{\mathbf{z}_{+}^{2}}=H^{2}\left(T^{2}\right)$ (H-outer for two variables), implies that $[\varphi]_{S}=\left[[\varphi]_{\mathbf{z}_{+}^{2}}\right]_{S}=\left[H^{2}\left(T^{2}\right)\right]_{S}=[S]$, we get that $\varphi$ is outer. This was originally proved in [2]. A counter example to the converse of the above statement was provided in [8]. In [7], a necessary and sufficient condition was given for the equivalence of the properties for a function to be $H$-outer and outer. Thus, in Theorem 3.1, we cannot replace the condition (iii) by the requirement that $\varphi$ be outer.

We note that if $g \in H^{2}\left(T^{2}\right)$, then $g$ is of $S$-analytic type. One can ask whether one can improve Lemma 2.4 under the assumption that $g \in H^{2}\left(T^{2}\right)$. Let us observe that, with $h$ as in (2.5), we have

$$
g\left(e^{i \lambda}, e^{i \mu}\right)=h\left(e^{i \lambda}\right)+e^{i \mu} \tilde{h}\left(e^{i \lambda}, e^{i \mu}\right),
$$

where $\tilde{h} \in H^{2}\left(T^{2}\right)$. If $g$ is outer, then, with $H^{2}\left(T_{\lambda}\right)=\overline{\operatorname{sp}}\left\{e^{i j \lambda}: j \in \mathbf{Z}_{+}\right\}$in $L^{2}\left(T^{2}, \sigma_{2}\right)$, we get

$$
H^{2}\left(T_{\lambda}\right) \subset[S]=[g]_{S}=\overline{\operatorname{sp}}\left\{e^{i j \lambda} h\left(e^{i \lambda}\right)\right\} \oplus M,
$$

with $M \perp H^{2}\left(T_{\lambda}\right)$. Since $h \in H^{2}\left(T_{\lambda}\right)$, we obtain

$$
H^{2}\left(T_{\lambda}\right)=\overline{\operatorname{sp}}\left\{e^{i j \lambda} h\left(e^{i \lambda}\right), j \geq 0\right\} .
$$

This shows that $h$ is $H$-outer in $T_{\lambda}$ or, equivalently, that $h$ is outer in $T$. In combination with Lemma 2.4 we obtain the following result from [5], with a simple proof.

Theorem 3.3. Let $g \in H^{2}\left(T^{2}\right)$; then $g$ is outer if and only if $h$, defined in (2.5) is outer in $T$, and $g$ is $\lambda$-outer.

Remark 3.4. One can derive other results in [5] with simple variations of the above arguments.

\section{REFERENCES}

1. S. A. Chobanyan and N. N. Vakhaniya, The Linear prediction and approximation of weak second order random elements. Prediction Theory and Harmonic Analysis: The Pesi Masani Volume (V. Mandrekar and H. Salehi, eds.), 37-60, North-Holland, Amsterdam, 1983.

2. H. Helson, Lectures on invariant subspaces. Academic Press, New York, 1964.

3. H. Helson and D. Lowdenslager, Prediction theory and Fourier series in several variables. Acta Math. 99(1958), 165-202. 
4. P. Ghatage and V. Mandrekar, On Beurling type invariant subspaces of $L^{2}\left(T^{2}\right)$ and their equivalence. J. Operator Theory 20(1988), 83-89.

5. K. Izuchi and Y. Matsugu, Outer functions and invariant subspaces on the torus. Acta Sci. Math. (Szeged) 59(1994), 429-440.

6. G. Kallianpur and V. Mandrekar, Non-deterministic random fields and Wold and Halmos decomposition for commuting isometries. Prediction Theory and Harmonic Analysis: The Pesi Masani Volume (V. Mandrekar and H. Salehi, eds.), 165-190, NorthHolland, Amsterdam, 1983.

7. V. Mandrekar, On the validity of Beurling theorems in polydiscs. Proc. Amer. Math. Soc. 103(1988), 145-148.

8. W. Rudin, Invariant subspaces of $H^{2}$ on a torus. J. Funct. Anal. 61(1985), 378-384.

9. A. R. Soltani, Extrapolation and moving average representation for stationary random fields and Beurling's theorem. Ann. Probab. 12(1984), 120-132.

(Received 3.07.2000)

Author's addresses:

L. Gawarecki and P. Richard

Kettering University

Department of Science and Mathematics

$1700 \mathrm{~W}$. Third Ave.

Flint, MI 48504, U.S.A.

E-mail: lgawarec@kettering.edu

prichard@kettering.edu

V. Mandrekar

Department of Statistics and Probability

Michigan State University

Wells Hall, East Lansing, MI 48840, U.S.A.

E-mail: mandrekar@stt.msu.edu 